\title{
Variants in the PNPLA1 Gene in Families with Autosomal Recessive Congenital Ichthyosis Reveal Clinical Significance
}

\author{
Farooq Ahmad a, b Ishtiaq Ahmed ${ }^{c}$ Qamre Alam $^{\text {d }}$ Tanveer Ahmad $^{\mathrm{e}}$ \\ Ammara Khan $^{\text {a }}$ ljaz Ahmad ${ }^{b}$ Muhammad Bilal $^{b}$ Amir Hayat $^{\dagger}$ \\ Amjad Khan $^{g}$ Ahmed Waqas ${ }^{h}$ Misbahuddin M. Rafeeq ${ }^{i}$ Ziaullah M. Sain $^{j}$ \\ Muhammad Umair ${ }^{\mathrm{d}, \mathrm{k}}$
}

\begin{abstract}
aDepartment of Chemistry, Women University Swabi, Swabi, Pakistan; 'bepartment of Biochemistry, Faculty of Biological Sciences, Quaid-i-Azam University (QAU), Islamabad, Pakistan; 'Mardan Medical Complex (MMC), Mardan, Pakistan; ${ }^{d}$ Medical Genomics Research Department, King Abdullah International Medical Research Center (KAIMRC), King Saud bin Abdulaziz University for Health Science, Ministry of National Guard-Health Affairs (MNGHA), Riyadh, Saudi Arabia; eDepartment of Biotechnology, Faculty of Chemical and Life Sciences, Abdul Wali Khan University (AWKUM), Mardan, Pakistan; fDepartment Biochemistry, Abdul Wali Khan University, Mardan, Pakistan; 'Laboratoire d'ImmunoRhumatologie Moléculaire, Plateforme GENOMAX, INSERM UMR_S 1109, Faculté de Médecine, Fédération Hospitalo-Universitaire OMICARE, Fédération de Médecine Translationnelle de Strasbourg (FMTS), LabEx TRANSPLANTEX, Université de Strasbourg, Strasbourg, France; ' Division of Science and Technology, Department of Zoology, University of Education Lahore, Multan Campus, Multan, Pakistan; 'Department of Pharmacology, Faculty of Medicine, Rabigh, King Abduaziz University, Jeddah, Saudi Arabia; jDepartment of Microbiology, Faculty of Medicine, Rabigh, King Abduaziz University, Jeddah, Saudi Arabia; ${ }^{2}$ Department of Life Sciences, School of Science, University of Management and Technology (UMT), Lahore, Pakistan
\end{abstract}

\section{Keywords}

Whole-exome sequencing $\cdot$ Autosomal recessive congenital ichthyosis - PNPLA1 - Novel and recurrent variants .

Pakistani families

\begin{abstract}
The term autosomal recessive congenital ichthyosis (ARCI) is the subgroup of ichthyosis, which describes a highly heterogeneous group of genetic disorders of the skin characterized by cornification and defective keratinocytes differentiation associated with mutations in at least 14 genes including PNPLA1. To study the molecular basis of the Pakistani kindreds $(A$ and $B$ ) affected by $A R C l$, whole-exome sequencing
\end{abstract}

(WES) in the DNA samples of affected members was performed followed by Sanger sequencing of the candidate gene to hunt down the disease-causing sequence variant/s. WES data analysis led to the identification of a novel nonsense sequence variant (c.892C>T; p.Arg298*, family A) and a recurrent missense variant (c.102C>A; p.Asp34Glu, family $B)$ in PNPLA1 mapped to the ARCI locus in chromosome 6p21.31. Validation and cosegregation analysis of the variants in the remaining family members of the respective families were confirmed by Sanger sequencing. The current investigation expands the spectrum of PNPLA1 mutations and helps establish the proper clinico-genetic diagnosis and correct genotype-phenotype correlation. @ 2021 S. Karger AG, Basel karger@karger.com

(C) 2021 S. Karger AG, Basel

www.karger.com/msy

Karger ${ }^{\prime}=$
Correspondence to:

Farooq Ahmad, farooq.amazai@gmail.com

Muhammad Umair, khugoo4u@yahoo.com 


\section{Introduction}

Autosomal recessive congenital ichthyosis (ARCI) describes a group of clinically and genetically heterogeneous cornification disorders. ARCI results from functional defects in the epidermal barrier components including intercellular lipid barrier, the cornified cell envelope, and keratin or filaggrin degradation products that result in characteristic chronic, universal scaling, and roughness of the skin. In the majority of ARCI cases, xerosis, fissures, erythema, and occasional pruritus have also been reported. ARCI may be life-threatening specifically in the first year of life due to severe skin inflammation, hypothermia, and dehydration. ARCI can be inherited as an isolated trait or in the form of syndromes in combination with other abnormal manifestations and share prevalence rate less than 1/200.000 [Oji and Traupe, 2009].

The nonsyndromic form of ARCI is caused by diseasecausing mutations in the genes significantly involved in keratinocyte differentiation and epidermal barrier function including $A B C A 12$ (MIM 607800) [Lefévre et al., 2003], NIPAL4 (MIM 609383) [Lefévre et al., 2004], PNPLA1 (MIM 612121) [Grall et al., 2012; Ahmad et al., 2016], LIPN (MIM 613924) [Israeli et al., 2011], SDR9C7 (MIM 609769) [Shigehara et al., 2016], TGM1 (MIM, 190195) [Huber et al., 1995], CERS3 (MIM 615276) [Radner et al., 2013], ALOX12B (MIM 603741) [Jobard et al., 2002], ALOXE3 (MIM 607206) [Jobard et al., 2002], CASP14 (MIM 605848) [Kirchmeier et al., 2017], CYP4F22 (MIM 611495) [Lefévre et al., 2006], and SULT2B1 (MIM 604125) [Heinz et al., 2017], thus presenting a high degree of genetic heterogeneity. Mutations in these genes account for $80 \%$ of the patients suffering from ARCI [Fischer, 2009; Fischer and Traupe, 2014]. Ichthyosis vulgaris, an autosomal semi-dominant form of ichthyosis caused by mutations in the FLG gene (MIM 135940) in chromosome 1q21.3 [Oji and Traupe, 2009]. The syndromic form of ARCI has been described, and the genetic cause has been associated with ST14 (MIM 606797) [Basel-Vanagaite et al., 2007; Ahmad et al., 2018a], whereas SLC27A4 (MIM 604194) [Klar et al., 2009] has been associated with autosomal recessive Ichthyosis prematurity syndrome.

The current investigation was performed in 2 consanguineous Pakistani kindred (A and $\mathrm{B}$ ). Clinical and genetic investigation of the affected members confirmed the diagnosis of ARIC carrying a novel truncating (c.892C $>\mathrm{T}$; p.Arg298*) and a missense (c.102C >A; p.Asp34Glu) variant in the PNPLA1 gene confirmed by Sanger sequencing.

\section{Materials and Methods}

Human Subjects and Study Approval

The current families (A and B) with the typical clinical features of ARCI were identified at the remote area of Khyber Pakhtunkhwa (KPK) province of Pakistan. Detailed clinical evaluation of the affected individuals was performed by expert dermatologists at the local government hospital.

Family History, Blood Samples Collection, and Genomic DNA Isolation

Family history includes the clinical and medical history interviews, a physical examination, review of medical and marriage records, and pedigree construction and analysis to evaluate the exact transmission of the disease phenotype. After review of the clinical information, pictures and pedigrees, the expert panel (including geneticists and dermatologists) concluded that the available affected individuals in families A and B were likely affected by nonsyndromic ARCI. Blood samples were collected in EDTA tubes from the 3 affected (II-4, II-7, IV-3) and 6 unaffected (II-2, II-6, III-2, III-3, III-6, IV-4) individuals of family A (Fig. 1a), and 3 affected (IV-1, IV-4, IV-5) and 4 unaffected (III-2, III-3, IV-2, IV-3) individuals of family $\mathrm{B}$ and were subjected to genomic DNA isolation using standard procedures. Furthermore, nanodrop-based quantification of DNA was done for future use.

\section{Genetic Linkage Analysis}

Microsatellite marker-based genotyping was performed for this purpose as previously described [Ahmad et al., 2019]. For genotyping with microsatellite markers, genes were prioritized based on the observed clinical manifestations. For this purpose, a minimum of 4 to 5 markers were typed boarding the genes involved in causing ARCI including ALOX12B (MIM 603741) and ALOXE3 (MIM 607206) at 17p13.1, ABCA12 (MIM607800) at 2q35, CYP4F22 (MIM 611495) at 19p13.12, NIPAL4 (MIM 609383) at 5q33.3, SDR9C7 (MIM 609769) at 12q13.3, and SULT2B1 (MIM 604125) at 19q13.33. Microsatellite markers were PCR amplified against the genomic DNA of all the available members of the families $\mathrm{A}$ and $\mathrm{B}$. Amplified products were resolved on non-denaturing polyacrylamide gel, stained with ethidium bromide, and alleles were assigned visually.

\section{Whole-Exome Sequencing}

None of the families showed linkage on the genes selected for microsatellite marker-based genotyping, so whole-exome sequencing (WES) was carried out. DNA samples from affected member IV-3 of family A and IV-1 of family B were subjected to WES. Solution hybridization exome capture was carried out using the Roche NimbleGenSeqCap EZ Human Exome Library v2.0. Nucleotide sequences of the captured regions were generated on Illumina HiSeq and NextSeq 500 platforms, respectively. Resulting sequence reads (FASTQ files) were aligned to a human reference sequence (UCSC Genome Browser hg19, GRCh37) with the software Burrows-Wheeler Aligner [Li and Durbin, 2009]. For realignment of regions containing indels, recalibration of base qualities, and variant detection and calling, the Genome Analysis Toolkit was used [McKenna et al., 2010]. All sequence variants were annotated with SeattleSeq137. Exome sequence data were analyzed for homozygous variants based on occurrence in public variant databases [dbSNP, 1000 Genomes, Exome Variant Server (EVS), Exome Aggregation Consortium (ExAC)] in 100 unrelated Paki- 


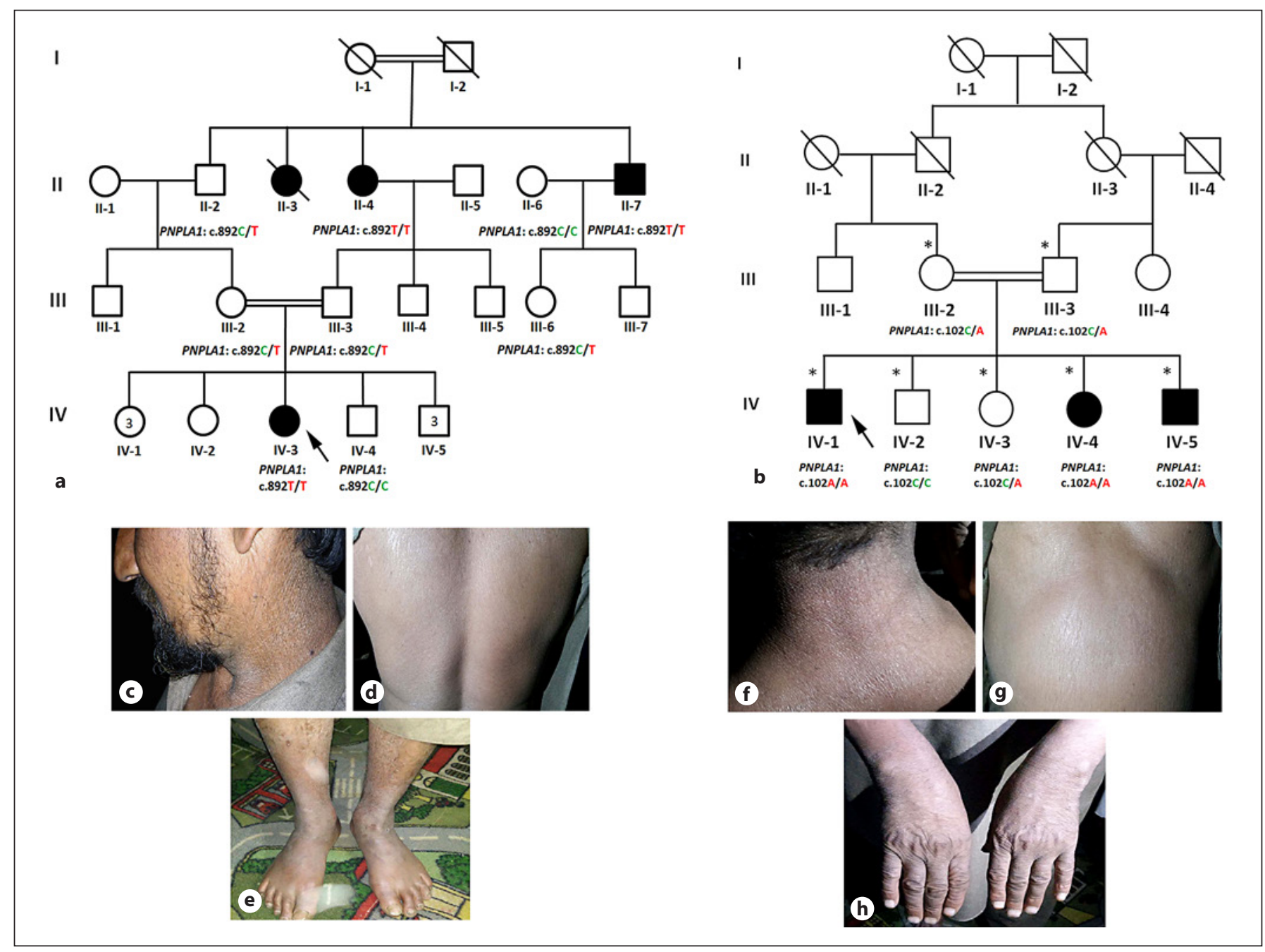

Fig. 1. a, b Pedigree of 2 extended Pakistani families affected by autosomal recessive congenital ichthyosis (ARCI). Symbolic representations in the pedigree: double lines (consanguineous unions), affected individuals (filled symbols), unaffected individuals (clear symbols), and diagonal line (deceased individuals). c-e Clinical photographs showing various parts of the body of affected individual of family A displaying the hallmarks of ARCI. c-e Ichthyotic features on face, neck, back and lower limbs. $\mathbf{f}-\mathbf{h}$ Clinical photographs of affected members of family B with scaly dry skin affecting the neck, belly, and hands.

stani control samples and in 215 in-house exome sequences from unrelated Pakistani individuals without ichthyosis.

Sanger Sequencing-Based Validation and Co-Segregation of Exome Identified Variants

The variants of interest underwent Sanger sequencing for validation and cosegregation analysis. Reference genomic sequence of PNPLA1 (NM_001145717) was obtained from the University of California Santa Cruz genome database browser (https://genome. ucsc.edu/cgi-bin/hgGateway). Two pairs of primers (available on request) were designed using online primer3 software (http:// primer3.ut.ee/) to sequence the variants and its flanking region. PCR-based amplification of the PNPLA1 gene was carried out using DNA samples of the available members of both families. Pro- cedure for the purification of the amplified products, Sanger sequencing and data analysis were performed as described by Ahmad et al. [2018b].

\section{In-silico Analysis}

To evaluate the effect of the variants, various online protein prediction tools were used including MutationTaster (http://www.mutationtaster.org/), SIFT (https://sift.bii.a-star.edu.sg/), and PolyPhen-2 (http://genetics.bwh.harvard.edu/pph2/), and also CADD scores were calculated for both the variants. Additionally, public databases like dbSNPs, 1000 genome, ExAC (http://exac.broadinstitute.org), gnomAD (https://gnomad.broadinstitute.org/), and 215 in-house exomes data of Pakistani individuals (HMG) were used to exclude the nonpathogenic nature of the variants. 


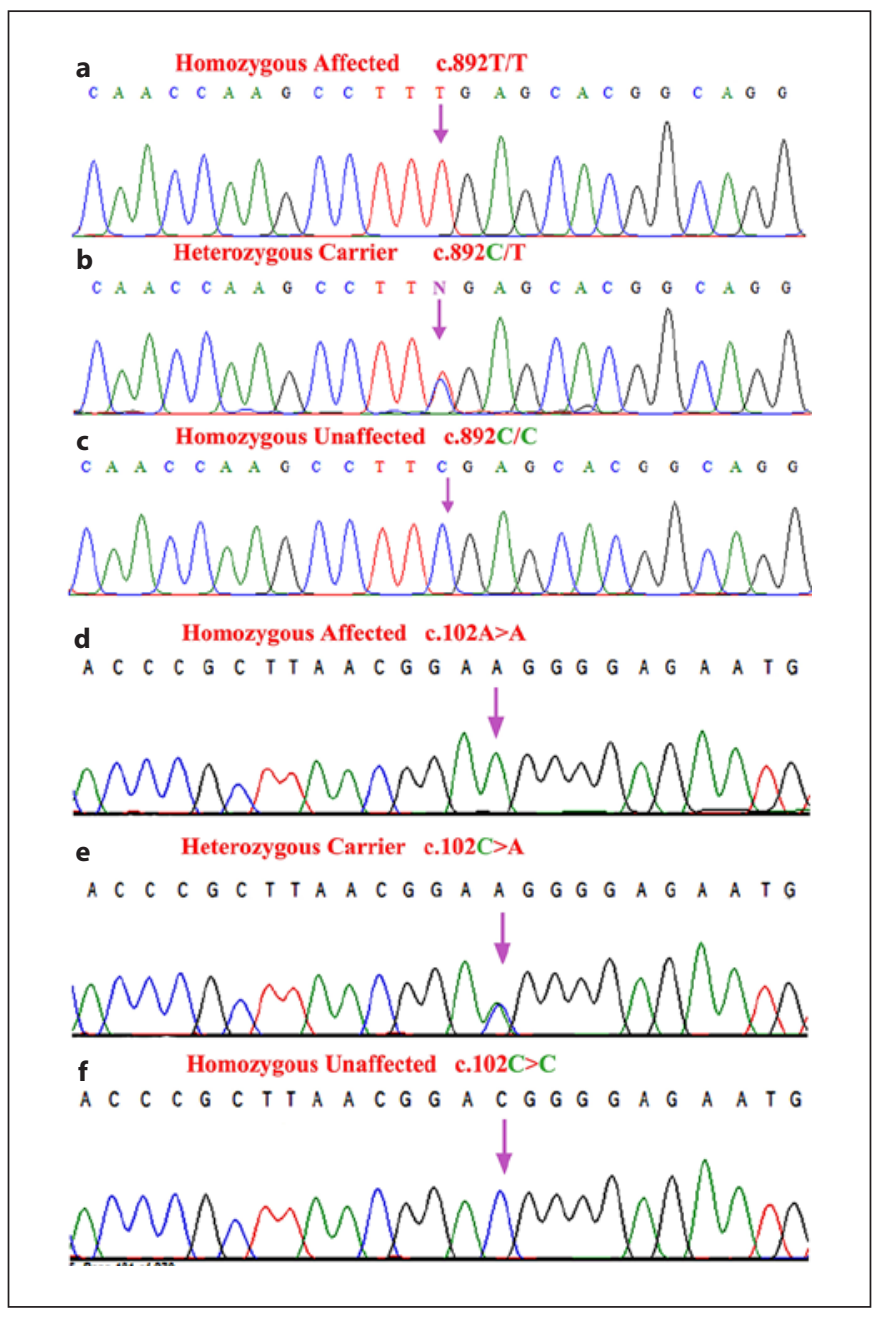

Fig. 2. a-c Sequence characteristics of PNPLA1 novel (c.892C>T; p.Arg $298^{*}$ ) sequence variant. Nucleotide sequence in the affected individual is shown in the upper panel (a), a heterozygous carrier in the middle panel (b), homozygous unaffected individual in the lower panel (c). The same pattern of segregation for the recurrent (c.102C > A p.Asp34Glu) sequence variant is presented in panel df. The arrows indicate the exact point of the mutations.

\section{Results}

\section{Clinical Profile}

The affected members in the family A and B were born to unaffected parents without any serious complications during pregnancies. They were born with standard birth weight without any signs of ectropion, eclabium, or collodion membrane. Typical clinical hallmarks of nonsyndromic ARCI were observed in the affected individuals of both families. Clinically, marked ichthyotic features were observed affecting the entire cutaneous surface (Fig. 1ch). At birth, affected members exhibited mild diffused scales on the body. Extreme dryness of the skin was observed on the extremities and other parts of the body due to minimal excretion of sweat (Fig. 1c, f, e, h). Severe ichthyotic features in form of dark brown scales were observed affecting the neck, chest, and lower limbs in the affected members of family A (Fig. 1c-e), whereas in family $B$, mild and diffused scales were observed affecting various regions of the body (Fig. 1f-h). Skin texture appeared hard, thick, and rough. Affected members had healthy nails, teeth, and hair. No signs or symptoms of any systemic abnormalities were observed. Intellectual disability, hyperkeratosis, epidermolysis bullosa were not observed.

Whole-Exome and Sanger Sequencing Data Analysis

WES data of affected member IV-3 of family A and IV-1 of family B were carefully analyzed for the variants of interest. Keeping in view the consanguinity and the inheritance pattern of the disease which is consistent with autosomal recessive in both the pedigrees, WES data were searched for the homozygous variants. This approach led to the identification of a novel PNPLA1 biallelic nonsense sequence variant (c.892C > T; p.Arg298*) in the affected member of family $\mathrm{A}$ and a previously known missense sequence variant (c.102C $>$ A; p.Asp34Glu) [Ahmad et al., $2016]$ in family $B$ in the same gene. The identified variants were subjected to validation and cosegregation analysis by Sanger sequencing. Analysis of the data obtained from the Sanger sequencing confirmed the validation and segregation of both the variants in their respective families (Fig. 2a-c: Family A; Fig. 2d-f: Family B).

In silico Analysis of the Novel (p.Arg298*) and

Recurrent (p.Asp34Glu) Variants

The predicted pathogenic effect of the novel ( $\mathrm{p}$. Arg298*) and recurrent (p.Asp34Glu) PNPLA1 variants were evaluated by various online protein prediction tools including SIFT and MutationTaster claiming it probably damaging and disease causing. CADD Phred scores for the novel and recurrent variants were calculated which are 35 and 8.554, respectively. Furthermore, checking of the novel variant across multiple online public databases such as dbSNPs, 1000 genomes, and 215 in-house data exomes of Pakistani individuals confirmed absence of the variant. Interestingly, the novel variant (p.Arg298*) was reported 4 times in heterozygote state (out of 121,362 alleles) in the ExAC database with an allelic frequency of 0.000003296 , whereas the homozygote state of the variant was found absent in the ExAC database. Additionally, the Arg298 and Asp34 were found highly conserved across different species (Fig. 3d,e), revealed by Clustal Omega W. 
Fig. 3. a Schematic representation of the coding region of the PNPLA1 gene with $\mathbf{b}$ known protein structural and functional domains of the gene product and localization of the novel and recurrent variants. The novel nonsense variant (c.892C $>\mathrm{T}$; p.Arg298*) and the recurrent missense variant (c.102C >A; p.Asp34Glu) identified in the present report are indicated by red bold writing affecting the exons 6 and 2 of the PNPLA1 coding transcript, respectively. c Representation of the truncated PNPLA1 protein as a result of mutation (p.Arg298*). Conservation of the Arg298 (d) and Asp34 (e) affected by mutation. The arrowheads indicate a conserved Arginine and Aspartate residue across different species. The intronic and exonic regions are not drawn to scale.

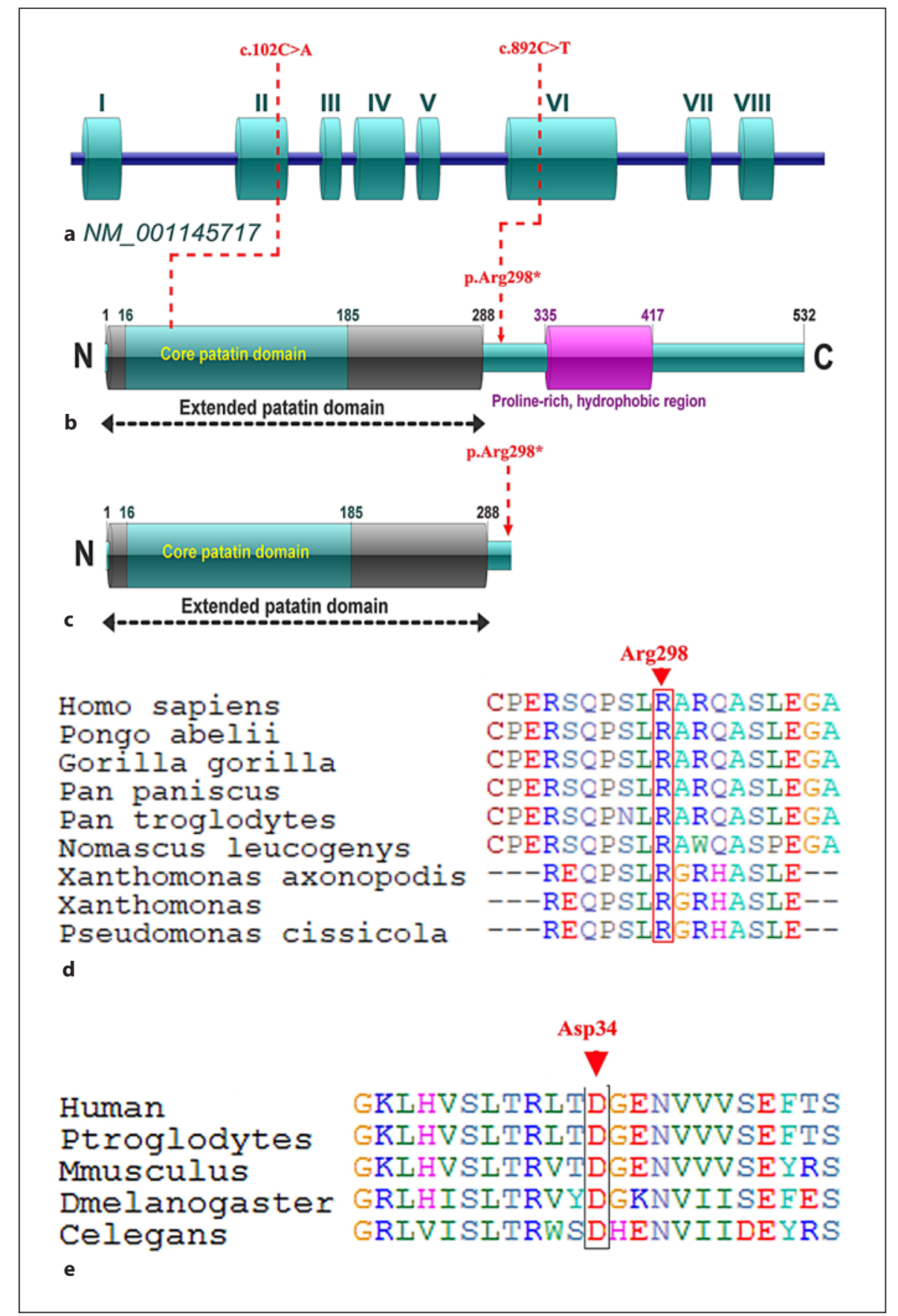

\section{Discussion}

In the current investigation, we added 2 consanguineous Pakistani lineal sampled from the remote area of KPK province of the country. Clinico-genetic study confirmed the involvement of a novel and a recurrent variant in the PNPLA1 gene, a previously known gene in the pathogenesis of ARCI10. Study design included linkage analysis with microsatellite markers followed by exome sequencing and Sanger sequencing of the PNPLA1 gene. Sequencing analysis of the PNPIA1 gene revealed a novel homozygous nonsense variant (c.892C>T; p.Arg298*) and a previously reported missense variant (p.Asp34Glu) [Ahmad et al., 2016] segregating in families A and B, respectively.

ARCI10 is a rare autosomal recessive disorder was reported for the first time by Grall et al. [2012] in a family 
Table 1. List PNPLA1 Mutation known to-date

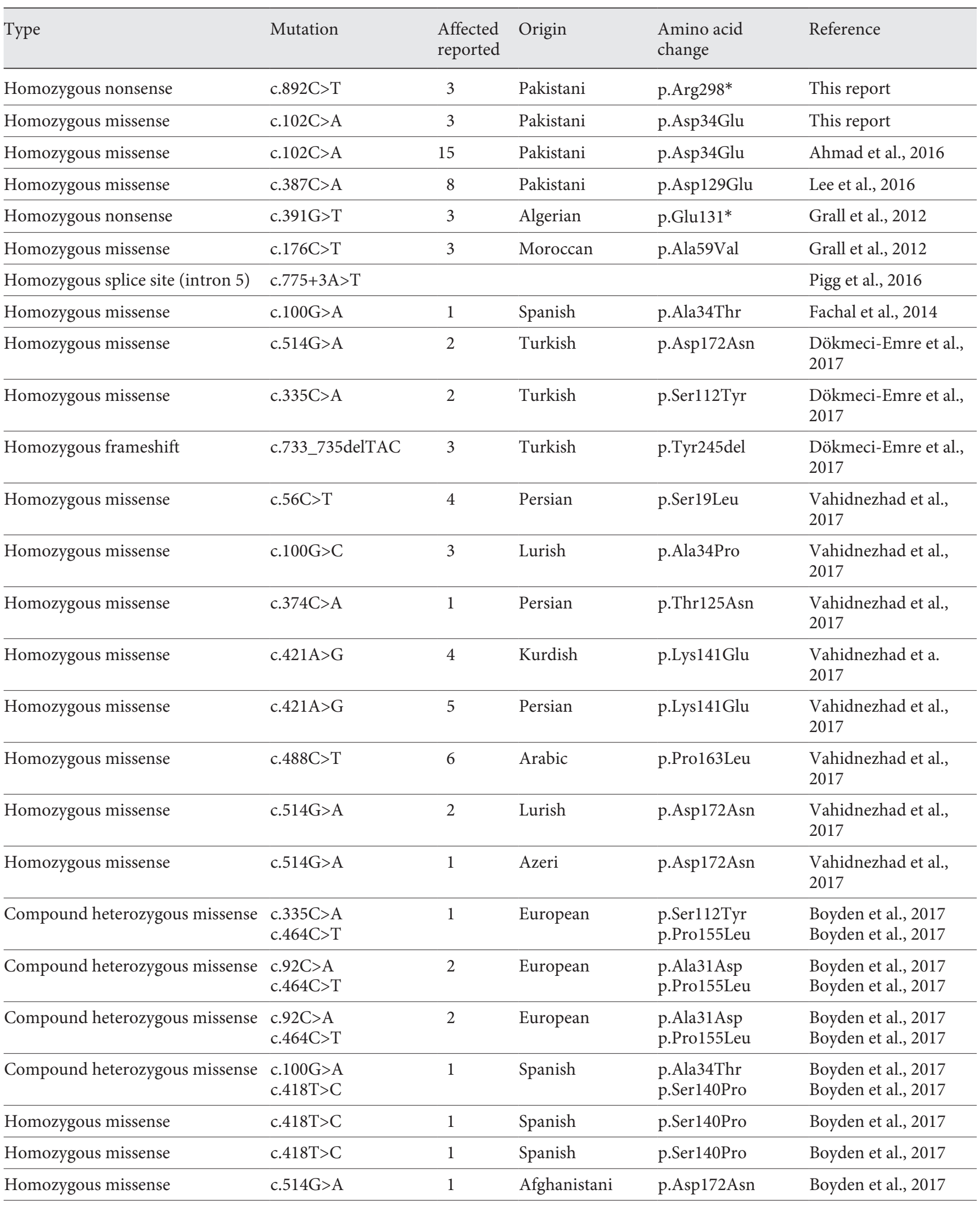


Table 1 (continued)

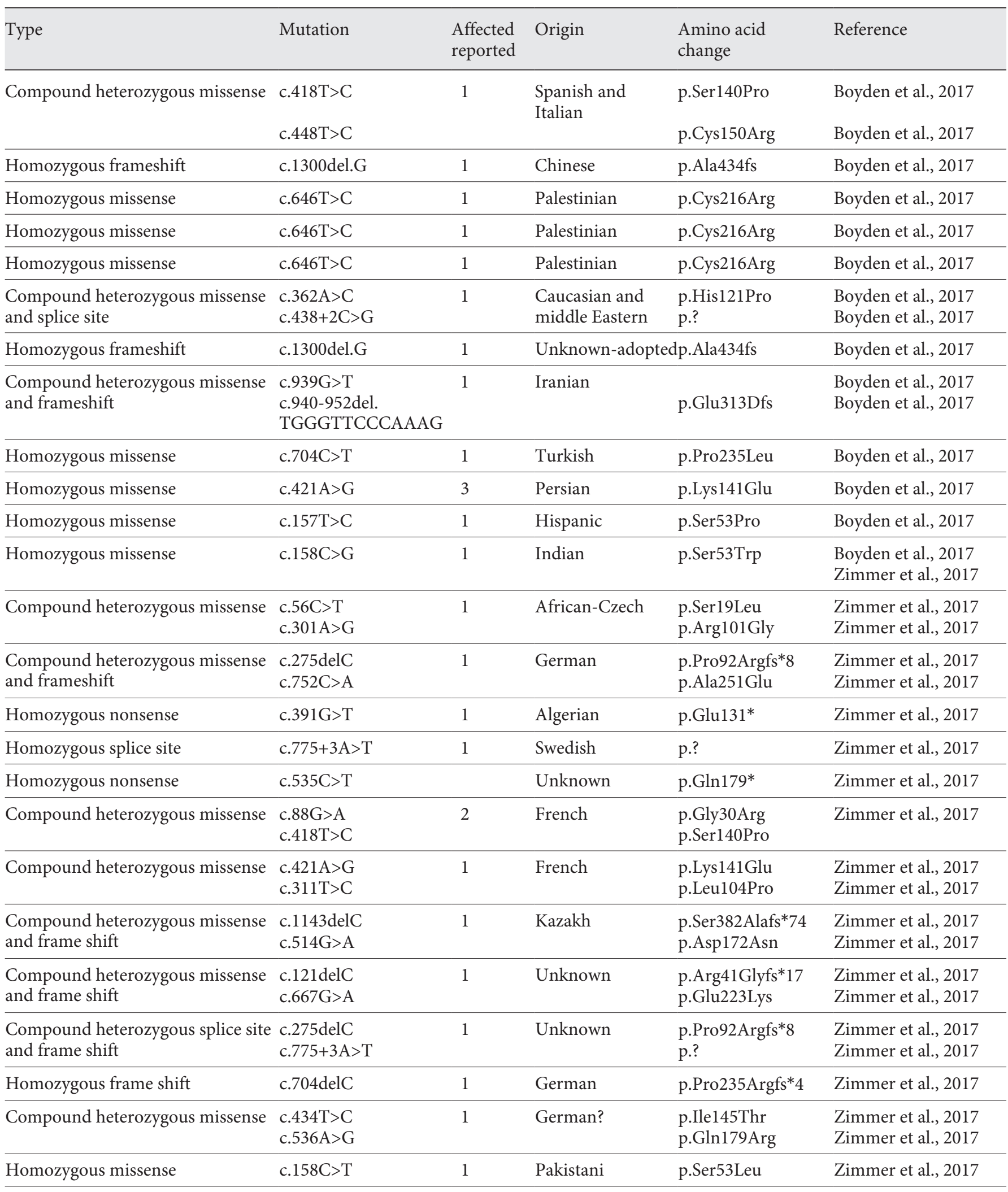


Table 1 (continued)

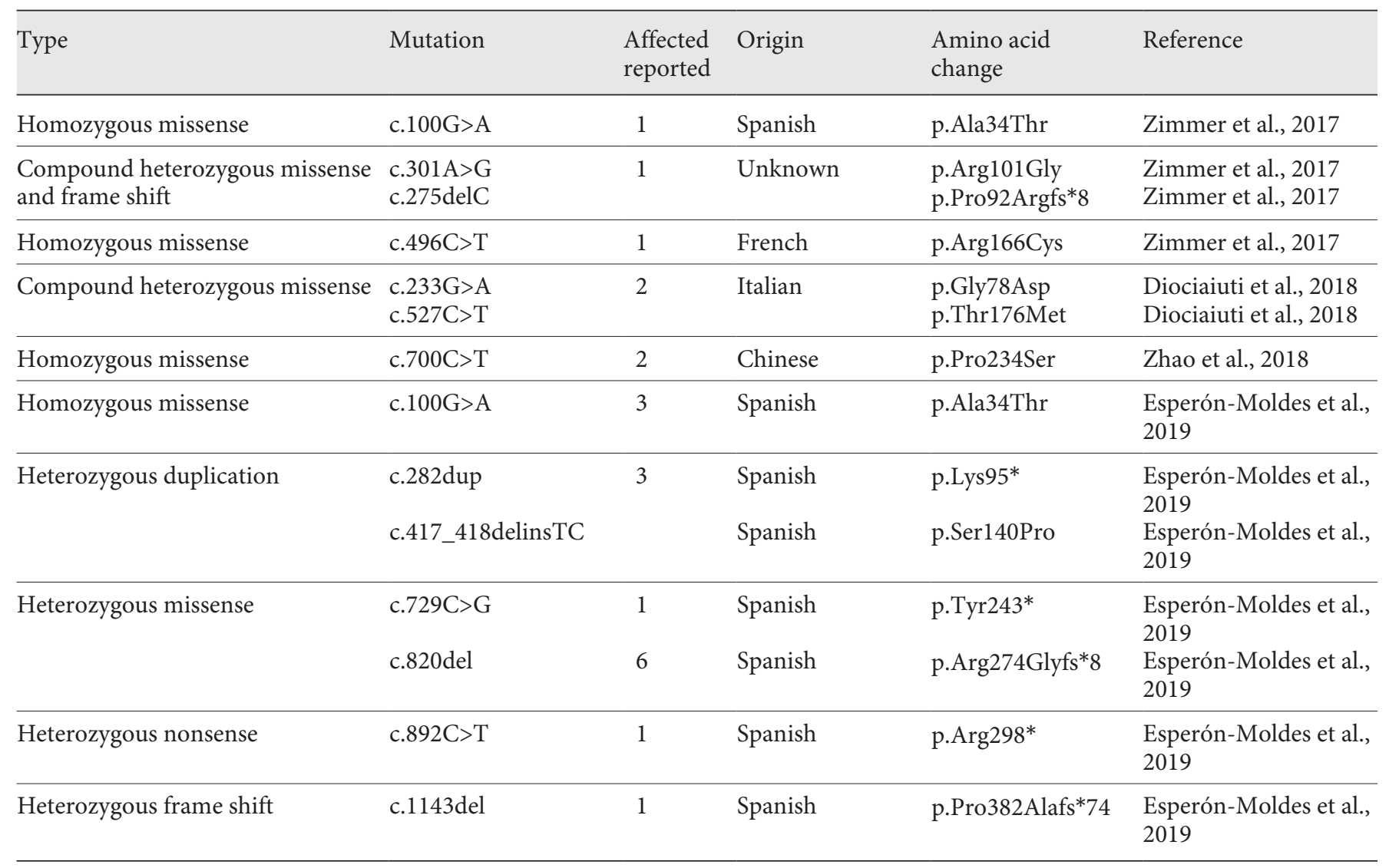

Table 2. Clinical features observed in the patients carrying a PNPLA1 mutation

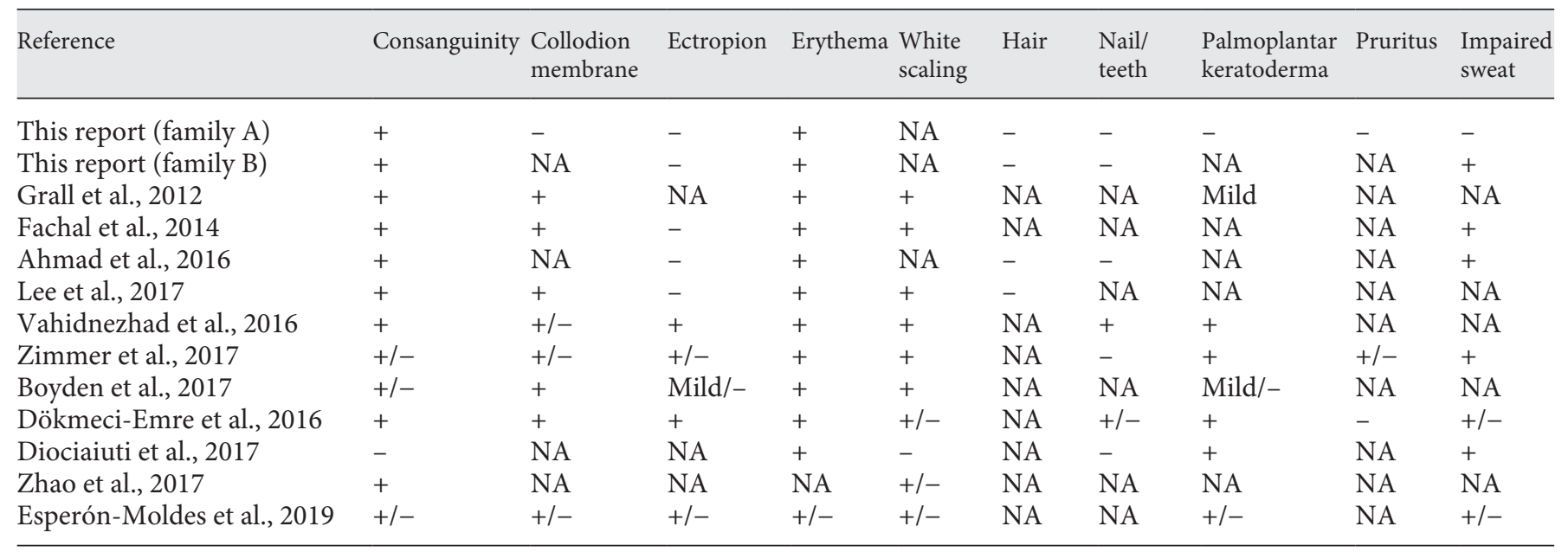

NA, not available. +, present; -, absent, +/-, present or absent. 
from Algerian and Moroccan origin caused by mutations in the PNPLA1 gene on chromosome 6p21.31, characterized by collodion membranes, erythema, mild palmoplantar keratoderma, mild pruritus, mild to moderate whitegrey scaling covering the entire cutaneous surface and flexures. Involvement of hair, nail, teeth, hypohidrosis and atopic dermatitis was not observed in the patient carrying PNPLA1 mutations. Later on Fachal et al. [2014] reported aSpanish family carrying sequence alteration in the PNPLA1 gene. For the first time Ahmad et al. [2016] reported three families of Pakistani origin with sequence variant in the PNPLA1 gene. The three families reported by Ahmad et al. [2016] represented Pakistani regions and no founder effect was found. The clinical synopsis presents ARCI typical hallmarks (Table 2). Later, several families were reported with mutations in the PNPLA1 gene. The details of the families, clinical features, and mutations are given in Table 1 and Table 2, respectively. To date, numbers of mutation have been reported in the PNPLA1 gene in families of different ethnicities. These include homozygous missense, nonsense, frameshift, splice site, and compound heterozygous mutations (Table 1). Most of the mutations reported previously affect the conserved patatin domain, whereas only 5 mutations [(p.Glu223Lys (P10), p.Ala251Glu (P2), p.Pro235Argfs*4 (P12), Pro259Serfs*2 (P4), p.Ser382Alafs*74 (P8)] [Zimmer et al., 2017], and the one reported here [(p. Arg298*), present report] lies outside the main patatin core domain (Fig. 3b). Clinically, variable combinations of ichthyotic features were observed in the patients reported by Zimmer et al. [2017] including collodion baby, fine lamellar ichthyosis, mild erythroderma, and white scaling. Brown and dry scaling skin, hyperlinearity, palmoplantar keratoderma, and impaired sweating were reported in some patients. Clinical synopsis of the patients of the present report (family A) resembles the clinical report of the P2, P4, P8, P10, and P12 reported by Zimmer et al. [2017]. Clinically, our patients exhibit mild and diffused scaling over the entire cutaneous surface. Scales were more prominent, dark brown in color, affecting the lower back, neck, belly, chest, lower limbs, and forehead. Mild erythema and severe dryness of the skin were also observed. No other systemic or ectodermal derived appendageal hallmarks were seen. In all the patients carrying mutations in the PNPLAl gene reported to date, considerable variability in the severity of the disease was noted including highly variable ichthyotic, palmoplantar keratoderma features, etc (Table 2). Similarly, variations in the phenotypic spectrum were also observed in the members of the same family with the same mutation, thus suggestive of the involvement of modifying genetic and environmental factors.

Novel and Recurrent PNPLA1 Mutations
PNPLA is a mammalian protein family consisting of 9 members divided into 3 subgroups: the first subgroup includes PNPLA1-PNPLA5, whereas mutationsin PNPLA2 and PNPLA3 have been associated with neutral lipid storage disease with myopathy and susceptibility to nonalcoholic fatty liver disease, respectively [Fischer et al., 2007; Romeo et al., 2008; Tian et al., 2010]. The subgroup II, called neuropathy target esterases, consists of PNPLA6 and PNPLA7, and the subgroup III, called phospholipases, contains PNPLA8 and PNPLA9. Members of this family share a highly conserved patatin domain and are involved in lipid metabolism and signal pathway, and exhibit diverse lipolytic and acyltransferase activities [Lake et al., 2005; Wilson et al., 2006].

The protein encoded by the PNPLA1 gene shares a highly conserved patatin-like domain (amino acids 16185). Most of the reported mutations in PNPLA1 to date reside in this particular domain, except for the 5 mutations (p.Glu223Lys; p.Ala251Glu; p.Pro235Argfs*4; Pro259Serfs*2; p.Ser382Alafs*74) [Zimmer et al., 2017] and the one reported here (p.Arg298*), thus attest the significance of PNPLA1 primary sequence particularly the core patatin domain in maintaining the proper function of this protein. The precise function of the protein is unknown but possesses a high degree of similarity with PNPLA2 having triglyceride hydrolase activity. Pnpla1knockout mice, wild type, and PNPLA1-deficient keratinocytes studies revealed its pivotal role in epidermal barrier maintenance and proper functioning [Grall et al., 2012; Grond et al., 2017; Pichery et al., 2017].Furthermore, Pnpla1-mutant knockout mice revealed that supplementation of ichthyotic skin with omega-O-acylceramides might be a therapeutic approach for treating skin symptoms in individuals affected by omega-O-acylceramide deficiency [Grond et al., 2017].

In conclusion, the patients described in this study harbored a novel nonsense mutation (p.Arg298*) in family $A$ and a previously known missense variant (c.102C $>A$; p.Asp34Glu) in family B. The novel nonsense mutation is predicted to cause truncation of the protein by 234 amino acids lacking the $\mathrm{C}$-terminus region, including the critical proline-rich hydrophobic region (Fig. 3c). As a result of truncation due to identified mutation, the protein might be unstable leading to nonsense mRNA-mediated decay that might result in the nullification of the PNPLA1 protein. Furthermore, the missense variant is predicted to cause local perturbation in the protein structure that might lead to protein misfolding and destabilization, thus results in particulate clinical pathology in the form of autosomal recessive congenital ichthyosis. 


\section{Acknowledgments}

We thank all the family members for their invaluable cooperation and participation in the present study.

\section{Statement of Ethics}

The study was approved by the institution IRB of the Women University Swabi Khyber Pakhtunkhwa Pakistan and followed standard Helsinki declarations. Detailed clinical evaluation of the affected individuals was performed by expert dermatologists at the local government hospital. Patients were enrolled with written parental consent for participation in the study, publication of case reports, and photographs.

\section{Conflict of Interest Statement}

The authors declare no conflicts of interest.

\section{Funding Sources}

This work was supported by the Higher Education Commission (HEC) Islamabad, Pakistan under start-up research grant program (SRGP) project \#2561.

\section{Author Contributions}

Farooq Ahmad and Muhammad Umair drafted the manuscript. Farooq Ahmad, Muhammad Bilal, Qamre Alam, Ammara Khan, Amjad Khan, Tanveer Ahmad, Ishtiaq Ahmed, Ijaz Ahmad, Amir Hayat, Ahmad Waqas collected samples, clinical data, analyzed data, and performed experiments. Misbahuddin M. Rafeeq and Ziaullah M. Sain performed data analysis. Conception and research study design by Farooq Ahmad and Muhammad Umair.

\section{References}

Ahmad F, Ansar M, Mehmood S, Izoduwa A, Lee $\mathrm{K}$, Nasir A, et al. A novel missense variant in the PNPLA1 gene underlies congenital ichthyosis in three consanguineous families. J Eur Acad Dermatol Venereol. 2016;30(12): e210-e213.

Ahmad F, Ahmed I, Nasir A, Umair M, Shahzad S, Muhammad D, et al. A disease-causing novel missense mutation in the ST14 gene underlies autosomal recessive ichthyosis with hypotrichosis syndrome in a consanguineous family. Eur J Dermatol. 2018a;28(2):209-16.

Ahmad F, Nasir A, Thiele H, Umair M, Borck G, Ahmad W, et al. A novel homozygous missense variant in NECTIN4 (PVRL4) causing ectodermal dysplasia cutaneous syndactyly syndrome. Ann Hum Genet. 2018b;82(4): 232-8.

Ahmad F, Ahmad T, Umair M, Abdullah, Ahmad W. Sequence variants in the EDAR gene causing hypohidrotic ectodermal dysplasia. Congenit Anom (Kyoto). 2019;59(4):145-7.

Basel-Vanagaite L, Attia R, Ishida-Yamamoto A, Rainshtein L, Ben Amitai D, Lurie R, et al. Autosomal Recessive Ichthyosis with Hypotrichosis Caused by a Mutation in ST14, Encoding Type II Transmembrane Serine Protease Matriptase. Am J Hum Genet. 2007;80(3): 467-77.

Boyden LM, Craiglow BG, Hu RH, Zhou J, Browning J, Eichenfield L, et al. Phenotypic spectrum of autosomal recessive congenital ichthyosis due to PNPLA1 mutation. Br J Dermatol. 2017;177(1):319-22.

Diociaiuti A, Pisaneschi E, Zambruno G, Angioni A, Novelli A, Boldrini R, et al. Novel PNPLA1 mutations in two Italian siblings with autosomal recessive congenital ichthyosis. J Eur Acad Dermatol Venereol. 2018;32(3):e110e112.
Dökmeci-Emre S, Taşkıran ZE, Yüzbaşığlu A, Önal G, Akarsu AN, Karaduman A, et al. Identification of two novel PNPLA1 mutations in Turkish families with autosomal recessive congenital ichthyosis. Turk J Pediatr. 2017;59(4):475-82.

Esperón-Moldes U, Ginarte Val M, RodríguezPazos L, Fachal L, Azaña JM, Barberá Fons M, et al. Novel and Recurrent PNPLA1 Mutations in Spanish Patients with Autosomal Recessive Congenital Ichthyosis; Evidence of a Founder Effect. Acta Derm Venereol. 2019; 99(10):894-8.

Fachal L, Rodríguez-Pazos L, Ginarte M, Carracedo A, Toribio J, Vega A. Identification of a novel PNPLA1 mutation in a Spanish family with autosomal recessive congenital ichthyosis. Br J Dermatol. 2014;170(4):980-2.

Fischer J. Autosomal recessive congenital ichthyosis. J Invest Dermatol. 2009;129(6):1319-21.

Fischer J, Traupe H. Klinik und Genetik der Ichthyosen. Medgen. 2014;26:427-42.

Fischer J, Lefèvre C, Morava E, Mussini JM, Laforêt $P$, Negre-Salvayre A, et al. The gene encoding adipose triglyceride lipase (PNPLA2) is mutated in neutral lipid storage disease with myopathy. Nat Genet. 2007;39(1):2830.

Grall A, Guaguère E, Planchais S, Grond S, Bourrat E, Hausser I, et al. PNPLA1 mutations cause autosomal recessive congenital ichthyosis in golden retriever dogs and humans. Nat Genet. 2012;44(2):140-7.

Grond S, Eichmann TO, Dubrac S, Kolb D, Schmuth M, Fischer J, et al. PNPLA1 Deficiency in Mice and Humans Leads to a Defect in the Synthesis of Omega-O-Acylceramides. J Invest Dermatol. 2017;137(2):394-402.

Heinz L, Kim GJ, Marrakchi S, Christiansen J, Turki H, Rauschendorf MA, et al. Mutations in SULT2B1 Cause Autosomal-Recessive Congenital Ichthyosis in Humans. Am J Hum Genet. 2017;100(6):926-39.

Huber M, Rettler I, Bernasconi K, Frenk E, Lavrijsen SP, Ponec M, et al. Mutations of keratinocyte transglutaminase in lamellar ichthyosis. Science. 1995;267(5197):525-8.

Israeli S, Khamaysi Z, Fuchs-Telem D, Nousbeck J, Bergman R, Sarig O, et al. A mutation in LIPN, encoding epidermal lipase $\mathrm{N}$, causes a late-onset form of autosomal-recessive congenital ichthyosis. Am J Hum Genet. 2011; 88(4):482-7.

Jobard F, Lefévre C, Karaduman A, Blanchet-Bardon C, Emre S, Weissenbach J, et al. Lipoxygenase-3 (ALOXE3) and 12(R)-lipoxygenase (ALOX12B) are mutated in non-bullous congenital ichthyosiformerythroderma (NCIE) linked to chromosome 17p13.1. Hum Mol Genet. 2002;11(1):107-13.

Kirchmeier P, Zimmer A, Bouadjar B, Rösler B, Fischer J. Whole-Exome-Sequencing Reveals Small Deletions in CASP14 in Patients with Autosomal Recessive Inherited Ichthyosis. Acta Derm Venereol. 2017;97(1):102-4.

Klar J, Schweiger M, Zimmerman R, Zechner R, $\mathrm{Li} \mathrm{H}$, Törmä H, et al. Mutations in the fatty acid transport protein 4 gene cause the ichthyosis prematurity syndrome. Am J Hum Genet. 2009;85(2):248-53.

Lake AC, Sun Y, Li JL, Kim JE, Johnson JW, Li D, et al. Expression, regulation, and triglyceride hydrolase activity of Adiponutrin family members. J Lipid Res. 2005;46(11):2477-87.

Lee E, Rahman OU, Khan MT, Wadood A, Naeem M, Kang C, et al. Whole exome analysis reveals a novel missense PNPLA1 variant that causes autosomal recessive congenital ichthyosis in a Pakistani family. J Dermatol Sci. 2016;82(1):46-8. 
Lefévre C, Audebert S, Jobard F, Bouadjar B, Lakhdar $\mathrm{H}$, Boughdene-Stambouli $\mathrm{O}$, et al. Mutations in the transporter $\mathrm{ABCA} 12$ are associated with lamellar ichthyosis type 2 . Hum Mol Genet. 2003;12(18):2369-78.

Lefévre C, Bouadjar B, Karaduman A, Jobard F, Saker S, Ozguc M, et al. Mutations in ichthyin a new gene on chromosome $5 \mathrm{q} 33$ in a new form of autosomal recessive congenital ichthyosis. Hum Mol Genet. 2004;13(20):247382.

Lefévre C, Bouadjar B, Ferrand V, Tadini G, Mégarbané $\mathrm{A}$, Lathrop $\mathrm{M}$, et al. Mutations in a new cytochrome P450 gene in lamellar ichthyosis type 3. Hum Mol Genet. 2006;5(5): 767-76.

Li H, Durbin R. Fast and accurate short read alignment with Burrows-Wheeler transform. Bioinformatics. 2009;25:1754-60.

McKenna A, Hanna M, Banks E, Sivachenko A, Cibulskis K, Kernytsky A, et al. The genome analysis toolkit: a Map Reduce framework for analyzing next-generation DNA sequencing data. Genome Res. 2010;20:1297-303.

Oji V, Traupe H. Ichthyosis: clinical manifestations and practical treatment options. Am J Clin Dermatol. 2009;10(6):351-64.
Pichery M, Huchenq A, Sandhoff R, SeverinoFreire M, Zaafouri S, Opálka L, et al. 113 PNPLA1 defects in patients with ichthyosis and KO mice unveil PNPLA1 irreplaceable function in epidermal omega-acylceramide synthesis and skin permeability barrier. Hum Mol Genet. 2017;26(10):1787-1800.

Pigg MH, Bygum A, Gånemo A, Virtanen M, Brandrup F, Zimmer AD, et al. Spectrum of Autosomal Recessive Congenital Ichthyosis in Scandinavia: Clinical Characteristics and Novel and Recurrent Mutations in $132 \mathrm{~Pa}-$ tients. Acta Derm Venereol. 2016;96(7):932-

Radner FP, Marrakchi S, Kirchmeier P, Kim GJ, Ribierre F, Kamoun B, et al. Mutations in CERS3 cause autosomal recessive congenital ichthyosis in humans. PLoS Genet. 2013;9(6): e1003536.

Romeo S, Kozlitina J, Xing C, Pertsemlidis A, Cox $\mathrm{D}$, Pennacchio LA, et al. Genetic variation in PNPLA3 confers susceptibility to nonalcoholic fatty liver disease. Nat Genet. 2008; 40(12):1461-5.

Shigehara Y, Okuda S, Nemer G, Chedraoui A, Hayashi R, Bitar F, et al. Mutations in SDR9C7 gene encoding an enzyme for vitamin A metabolism underlie autosomal recessive congenital ichthyosis. Hum Mol Genet. 2016; 25(20):4484-93.
Tian C, Stokowski RP, Kershenobich D, Ballinger DG, Hinds DA. Variant in PNPLA3 is associated with alcoholic liver disease. Nat Genet. 2010;42(1):21-3.

Vahidnezhad H, Youssefian L, Saeidian AH, Zeinali S, Mansouri P, Sotoudeh S, et al. GeneTargeted Next Generation Sequencing Identifies PNPLA1 Mutations in Patients with a Phenotypic Spectrum of Autosomal Recessive Congenital Ichthyosis: The Impact of Consanguinity. J Invest Dermatol. 2017;137(3): 678-85.

Wilson PA, Gardner SD, Lambie NM, Commans SA, Crowther DJ. Characterization of the human patatin-like phospholipase family. J Lipid Res. 2006;47(9):1940-9.

Zhao HJ, Zeng X, Lei PC, Jiang XD, Li XM, Yan $\mathrm{HM}$, et al. A Chinese family with autosomal recessive congenital ichthyosis and Leber congenital amaurosis due to mutations in PNPLA1 and LCA5. Eur J Dermatol. 2018. Online ahead of print.

Zimmer AD, Kim GJ, Hotz A, Bourrat E, Hausser I, Has C, et al. Sixteen novel mutations in PNPLA1 in patients with autosomal recessive congenital ichthyosis reveal the importance of an extended patatin domain in PNPLA1 that is essential for proper human skin barrier function. Br J Dermatol. 2017;177(2):445-55. 